\title{
A PRIORI ERROR ESTIMATES FOR A STATE-CONSTRAINED ELLIPTIC OPTIMAL CONTROL PROBLEM
}

\author{
Arnd Rösch ${ }^{1}$ And SimeOn Steinig ${ }^{2}$
}

\begin{abstract}
We examine an elliptic optimal control problem with control and state constraints in $\mathbb{R}^{3}$. An improved error estimate of $\mathcal{O}\left(h^{s}\right)$ with $\frac{3}{4} \leq s \leq 1-\varepsilon$ is proven for a discretisation involving piecewise constant functions for the control and piecewise linear for the state. The derived order of convergence is illustrated by a numerical example.
\end{abstract}

Mathematics Subject Classification. 49J20, 35B45.

Received November 18, 2010. Revised October 5, 2011.

Published online February 13, 2012.

\section{INTRODUCTION}

In this paper we consider the optimal control problem

$$
\begin{gathered}
\min _{u \in L^{2}(\Omega)} J(y, u):=\frac{1}{2}\left\|y-y_{d}\right\|_{L^{2}(\Omega)}^{2}+\frac{\nu}{2}\|u\|_{L^{2}(\Omega)}^{2} \\
\text { s.t. } \\
-\Delta y=u \quad \text { in } \Omega \\
y=0 \quad \text { on } \Gamma=\partial \Omega \\
\text { and } \\
a \leq u(x) \leq b, \quad \text { a.e. in } \Omega \\
y(x) \geq y_{c}(x) \quad \text { in } \Omega,
\end{gathered}
$$

where $y_{c} \in C(\bar{\Omega})$ and $a, b \in \mathbb{R}, b>a . y_{d}$ is a given $L^{2}$-function, $\Omega$ is a convex bounded domain in $\mathbb{R}^{3}$ of class $C^{2}$. Throughout this paper we will refer to this problem as $(\mathrm{P})$.

Such elliptic optimal control problems have often been investigated in many of their facets, compare e.g. $[8,11,13,19,22,27]$, to name but a few. There have been discussions of regularity properties for solutions and Lagrange multipliers, of regularisation methods and of ways to solve such problems numerically. In particular, a priori estimates have been proven in $[12,13,16,18]$.

\footnotetext{
Keywords and phrases. Elliptic optimal control problem, state constraint, a priori error estimates.

1 Universität Duisburg-Essen, Fakultät für Mathematik, Forsthausweg 2, 47057 Duisburg, Germany. arnd.roesch@uni-due.de

2 Universität Stuttgart, Institut für Angewandte Analysis und Numerische Simulation, Pfaffenwaldring 57, 70569 Stuttgart,

Germany. simeon.steinig@ians.uni-stuttgart.de
} 
For the variational discretisation technique, where the control $u$ remains undiscretised, an a priori error estimate of $\mathcal{O}(h|\ln h|)$ was proven in [16]. However, for the fully discretised scheme, which we consider, the approximation results are restricted to $\mathcal{O}\left(h^{1 / 2}\right)$ proven in [13] and $\mathcal{O}\left(h^{1 / 2-\varepsilon}\right)$ shown in [18]. These were derived under the general result - compare e.g. [6] - that the solution $\bar{u}$ of problem (P) belongs to the space $W^{1, s}(\Omega)$ with $s<\frac{d}{d-1}$, $d$ being the dimension of the underlying domain $\Omega$.

However, using the very recent result [7], Theorem 4.3, where $H^{1}$-regularity for the optimal control $\bar{u}$ was established, we found a way to improve the convergence order of $h^{\frac{1}{2}-\varepsilon}$ : in this paper, we will obtain a rate of at least $h^{\frac{3}{4}}$. For higher regularity for the control $\bar{u}$ this can be improved to a rate of $h|\ln h|$ in case $\bar{u} \in W^{1, p}(\Omega)$, $p>3$.

We were also able to verify this convergence rate by a numerical example for a state-constrained optimal control problem on the unit ball $B_{1}(0)$ in $\mathbb{R}^{3}$. Our computations confirm the derived theoretical results: a convergence order of 'nearly' $h$ is demonstrated for a sufficiently regular optimal control $\bar{u}$.

The paper will be structured in the following fashion: in Section 2 we will briefly list some existence and regularity results for our optimal control problem, presenting also a necessary and sufficient optimality condition. We will introduce the discretisation of this problem, dwelling also very succinctly on optimality conditions for this discretisation in Section 3. In Section 4 we will present the general outline of the proof of the rate of convergence, highlighting the crucial steps that need to be taken. It will turn out that we need some approximation results, which we will prove in the ensuing sections. We will conclude this paper by finishing the proof of the rate of convergence and presenting the results of our numerical experiments.

\section{EXISTENCE AND OPTIMALITY}

It is well known that the governing PDE in (P) admits a unique weak solution $y \in H_{0}^{1}(\Omega)$ for every right-hand side $u \in L^{2}(\Omega)$. We define the control-to-state mapping $S: L^{2}(\Omega) \rightarrow L^{2}(\Omega)$ by $S: u \mapsto y$. Due to the regularity of the domain $\Omega$, it is also an element of $H^{2}(\Omega)$ and we additionally obtain:

$$
\|y\|_{H^{2}(\Omega)}=\|S u\|_{H^{2}(\Omega)} \leq c\|u\|_{L^{2}(\Omega)}
$$

for some generic constant $c$.

With the aid of this mapping $S$ we can now define the admissible set $U_{a d}$ for the optimal control problem:

$$
U_{a d}:=\left\{u \in L^{2}(\Omega): a \leq u(x) \leq b \text { a.e. in } \Omega,(S u)(x) \geq y_{c}(x) \text { in } \Omega\right\} .
$$

Let us at this stage point out that we have included the state constraint in $(\mathrm{P})$ into the set of admissible functions.

To do any meaningful optimisation we have to at least assume that $U_{a d}$ is non-empty. However, since this will not be enough for our subsequent analyses, we will immediately demand a stronger, Slater type assumption.

Assumption 2.1. There exists $u_{s} \in H^{1}(\Omega) \cap U_{a d}$ and $\tau \in \mathbb{R}, \tau>0$ so that

$$
\left(S u_{s}\right)(x) \geq y_{c}(x)+\tau
$$

for all $x \in \Omega$.

Remark 2.2. In contrast to weaker assumptions such as the one made in [20], Section 3.2.2, the assumption above might seem unduly strict. We will see, however, that our stronger assumption above immediately implies the existence of feasible points for the discretised problems for sufficiently small discretisation parameters $h$, compare Remark 3.1. It is not clear to the authors that such a generalised assumption as the one made in [20] guarantees the existence of feasible points for the discretised problems. This property, though, is essential for all discussions. 
For the subsequent standard existence, uniqueness and first-order optimality results we refer to [28].

Our optimal control problem admits a unique solution $\bar{u}$, after all $U_{a d}$ is bounded, convex and closed and thus weakly compact. For the unique optimal state we write $\bar{y}$ with $\bar{y}:=S \bar{u}$. Defining the adjoint state $\bar{p}$ by $\bar{p}=S^{*}\left(\bar{y}-y_{d}\right)$ - the star denotes the adjoint operator -, we can then write the first-order optimality condition in the following form:

$$
(\bar{p}+\nu \bar{u}, u-\bar{u}) \geq 0 \quad \forall u \in U_{a d} .
$$

Since $S$ is self-adjoint in $L^{2}(\Omega), \bar{p}$ can be equivalently expressed as $\bar{p}=S\left(\bar{y}-y_{d}\right)$. The adjoint state is thus defined analogously to the purely control-constrained case. This is a consequence of our multiplier-free approach: We have tucked the state constraint into the admissible set and thus avoid dealing with these irregular multipliers, which in general only belong to $L^{\infty}(\Omega)^{*}$, a space of measures.

The adjoint state can be equivalently characterised as the solution of a partial differential equation, namely, $\bar{p}$ is the unique (weak) solution of:

$$
\begin{aligned}
-\Delta \bar{p} & =\bar{y}-y_{d} \quad \text { in } \Omega \\
\bar{p} & =0 \quad \text { on } \Gamma .
\end{aligned}
$$

We remark briefly that $\bar{p} \in H^{2}(\Omega)$ because of the smooth boundary of $\Omega$. The following continuity property holds:

$$
\|\bar{p}\|_{H^{2}(\Omega)}=\left\|S\left(\bar{y}-y_{d}\right)\right\|_{H^{2}(\Omega)} \leq c\left(\|\bar{y}\|_{L^{2}(\Omega)}+\left\|y_{d}\right\|_{L^{2}(\Omega)}\right) .
$$

At the moment our solution $\bar{u}$ only belongs to $L^{2}(\Omega)$, or, to be more precise, to $L^{\infty}(\Omega)$ because of the box constraints on the control in $(\mathrm{P})$. However, as we have noted in the introduction, this is not the whole story. Thanks to the result in [7], Theorem 4.3, which is also valid for (local) optima for problems governed by semilinear elliptic equations, $\bar{u}$ is an $H^{1}(\Omega)$-function.

Let us now introduce the discretisation of this problem.

\section{Discretisation}

We triangulate our domain $\Omega$ with tetrahedra $T$. For the corresponding triangulation we write $\mathcal{T}_{h}$. The index $h$ denotes a suitable measure of the triangulation's fineness, i.e.: $h:=\sup _{T \in \mathcal{T}_{h}} \operatorname{diam}(T)$. The triangulation $\mathcal{T}_{h}$ of $\Omega$ with tetrahedra $T$ yields a polyhedral domain $\Omega_{h}=\bigcup_{T \in \mathcal{T}_{h}} T$ with $\Omega_{h} \subset \Omega$ because of the convexity of $\Omega$.

To establish error estimates for any finite element approximation of the PDE's solution, we need some regularity conditions for the sequence of triangulations $\left(\mathcal{T}_{h}\right)_{h>0}$ : we assume it to be conforming, shape regular and uniform. For details we refer to [10].

The control $u$ is discretised by piecewise constant functions, i.e. we replace $L^{2}(\Omega)$ in our original formulation of the problem $(\mathrm{P})$ by the space $U_{h}$ with

$$
U_{h}:=\left\{u \in L^{2}(\Omega):\left.u\right|_{T \in \mathcal{T}_{h}} \in \mathcal{P}_{0},\left.u\right|_{\Omega \backslash \Omega_{h}}=0\right\} .
$$

Of course, we have to discretise the state $y$, too. To do so, we choose standard linear finite element functions:

$$
Y_{h}:=\left\{y \in C(\bar{\Omega}):\left.y\right|_{T \in \mathcal{T}_{h}} \in \mathcal{P}_{1}(T),\left.y\right|_{\Gamma \cap\left(\Omega \backslash \Omega_{h}\right)}=0\right\} .
$$

We can now formulate the discrete analogon to the continuous problem $(\mathrm{P})$ :

$$
\begin{gathered}
\min _{u_{h} \in U_{h}} J(y, u):=\frac{1}{2}\left\|y_{h}-y_{d}\right\|_{L^{2}(\Omega)}^{2}+\frac{\nu}{2}\left\|u_{h}\right\|_{L^{2}(\Omega)}^{2} \\
\text { s.t. } \\
\left(\nabla y_{h}, \nabla v_{h}\right)_{L^{2}(\Omega)}=\left(u_{h}, v_{h}\right)_{L^{2}(\Omega)} \quad \forall v_{h} \in Y_{h} \\
\text { and } \\
a \leq u_{h}(x) \leq b, \quad \text { a.e. in } \Omega \\
y_{h}(x) \geq y_{c}(x) \quad \text { in } \Omega
\end{gathered}
$$


The existence of a unique optimal solution $\bar{u}_{h}$ can be deduced quite analogously to the continuous case. That there indeed exists a function in $U_{h}$ satisfying all the conditions in $\left(\mathrm{P}_{\mathrm{h}}\right)$ can be proven for a suitably fine mesh size, compare Lemma 6.2. Similarly, we can define a discrete control-to-state mapping $S_{h}$ as precisely the solution operator of the discretised PDE in $\left(\mathrm{P}_{\mathrm{h}}\right)$ by setting $S_{h} u_{h}:=y_{h}$. In particular, this yields a discrete optimal state $\bar{y}_{h}=S_{h} \bar{u}_{h}$. Furthermore, we can derive a discrete counterpart to (2.1). We introduce the discrete adjoint state $\bar{p}_{h}=S_{h}^{*}\left(y_{h}-y_{d}\right)$, then the first order optimality condition for $\left(\mathrm{P}_{\mathrm{h}}\right)$ can be written as:

$$
\left(\bar{p}_{h}+\nu \bar{u}_{h}, u_{h}-\bar{u}_{h}\right) \geq 0 \quad \forall u_{h} \in U_{a d}^{h},
$$

where $U_{h}$ is the discrete admissible set, i.e.

$$
U_{a d}^{h}:=\left\{u_{h} \in U_{h}: a \leq u_{h}(x) \leq b \text { a.e. in } \Omega, \quad\left(S_{h} u_{h}\right)(x) \geq y_{c}(x) \text { in } \Omega\right\} .
$$

Remark 3.1. If Assumption 2.1 is satisfied one can guarantee that $U_{a d}^{h}$ is non-empty for small enough $h$. The proof runs along the same lines as the one of Theorem 6.2.

At this stage it is convenient to list some approximation results. We will sum them up in a short lemma.

Lemma 3.2. Let $S$ and $S_{h}$ be given as above. Besides let $h_{d}$ be given such that $g(h):=h^{2}|\ln h|^{2}$ is monotonically decreasing for $0<h \leq h_{d}<1$. Then for the $C^{2}$-domain $\Omega$ the following error bounds hold true:

$$
\begin{aligned}
\left\|\left(S-S_{h}\right) u\right\|_{H^{1}(\Omega)} & \leq c h\|u\|_{L^{2}(\Omega)} \\
\left\|\left(S-S_{h}\right) u\right\|_{L^{2}(\Omega)} & \leq c h^{2}\|u\|_{L^{2}(\Omega)} \\
\left\|\left(S-S_{h}\right) u\right\|_{L^{\infty}(\Omega)} & \leq h^{2}|\ln h|^{2}\|u\|_{L^{\infty}(\Omega)}, \quad \forall h \leq h_{d},
\end{aligned}
$$

where $u$ is an arbitrary function in $L^{\infty}(\Omega)$.

For $u \in H^{1}(\Omega)$, the $L^{2}$-projection $P_{h}$ on the space of piecewise constant functions and $1 \leq p<\infty$ the following inequalities hold true:

$$
\begin{aligned}
\left\|u-P_{h} u\right\|_{L^{p}(\Omega)} & \leq c h\|u\|_{W^{1, p}(\Omega)} \\
\left\|u-P_{h} u\right\|_{W^{1, p^{\prime}}(\Omega)^{*}} & \leq c h^{2}\|u\|_{W^{1, p}(\Omega)},
\end{aligned}
$$

where $p^{\prime}$ denotes the dual exponent to $p$ with $\frac{1}{p}+\frac{1}{p^{\prime}}=1$, and $*$ the dual space.

Proof. The first two results in (3.2) are standard finite element approximation results (see e.g. [10]). The third can be found in [25], Theorem 5.1, where one additionally has to use that $S u \in W^{2, p}(\Omega) \cap W_{0}^{1, p}(\Omega)$ for all $p<\infty$ (compare [14], Thm. 2.4.2.5).

(3.3) is again a standard result, while for (3.4) we used the orthogonality property of the residual $u-P_{h} u \in U_{h}^{\perp}$.

\section{General outline for proving the Rate of COnvergence}

Let us recall the continuous and discrete optimality conditions (2.1) and (3.1).

$$
\begin{array}{rlrl}
(\bar{p}+\nu \bar{u}, u-\bar{u}) & \geq 0 & & \forall u \in U_{a d} \\
\left(\bar{p}_{h}+\nu \bar{u}_{h}, u_{h}-\bar{u}_{h}\right) \geq 0 & & \forall u_{h} \in U_{a d}^{h} .
\end{array}
$$

Now let us for the moment assume that we have constructed suitable test functions $u_{\delta}$ in $U_{a d}$ and $u_{\sigma}^{h} \in U_{a d}^{h}$ which are in some sense 'close' to $\bar{u}_{h}$ and $\bar{u}$ respectively, i.e. the distances $\left\|u_{\delta}-\bar{u}_{h}\right\|$ and $\left\|u_{\sigma}^{h}-\bar{u}\right\|$ are small. At this stage we do not specify the norms we use, as we need some room to 'manoeuvre' in this area. 
We can now test the above inequalities with the two functions $u_{\delta}$ and $u_{\sigma}^{h}$ and add them:

$$
\begin{aligned}
\left(\bar{p}+\nu \bar{u}, u_{\sigma}^{h}-\bar{u}\right)+\left(\bar{p}_{h}+\nu \bar{u}_{h}, u_{\delta}-\bar{u}_{h}\right)= & \underbrace{\left(\bar{p}+\nu \bar{u}, \bar{u}_{h}-\bar{u}\right)}_{=(*)}+\left(\bar{p}+\nu \bar{u}, u_{\sigma}^{h}-\bar{u}_{h}\right) \\
& +\underbrace{\left(\bar{p}_{h}+\nu \bar{u}_{h}, \bar{u}-\bar{u}_{h}\right)}_{=(* *)}+\left(\bar{p}_{h}+\nu \bar{u}_{h}, u_{\delta}-\bar{u}\right) \geq 0 .
\end{aligned}
$$

After a short computation we find that

$$
\begin{aligned}
(*) & =\left(\bar{p}, \bar{u}_{h}-\bar{u}\right)+\nu\left(\bar{u}, \bar{u}_{h}-\bar{u}\right) \\
(* *) & =\left(\bar{p}_{h}, \bar{u}-\bar{u}_{h}\right)+\nu\left(\bar{u}_{h}, \bar{u}-\bar{u}_{h}\right) .
\end{aligned}
$$

The addition of these two terms above and some further rearranging lead to the inequality:

$$
\nu\left\|\bar{u}-\bar{u}_{h}\right\|_{L^{2}(\Omega)}^{2} \leq\left(\bar{p}-\bar{p}_{h}, \bar{u}_{h}-\bar{u}\right)+\left(\bar{p}+\nu \bar{u}, u_{\sigma}^{h}-\bar{u}\right)+\left(\bar{p}_{h}+\nu \bar{u}_{h}, u_{\delta}-\bar{u}\right) .
$$

Using the definition of the adjoint state, we can estimate the first term on the right-hand side in the following fashion:

$$
\begin{aligned}
\left(\bar{p}-\bar{p}_{h}, \bar{u}_{h}-\bar{u}\right) & =\left(S^{*}\left(\bar{y}-y_{d}\right)-S_{h}^{*}\left(\bar{y}_{h}-y_{d}\right), \bar{u}_{h}-\bar{u}\right) \\
& =-\left\|S \bar{u}-S_{h} \bar{u}_{h}\right\|_{L^{2}(\Omega)}^{2}+\left(S \bar{u}-y_{d}, S \bar{u}_{h}-S_{h} \bar{u}_{h}\right)+\left(S_{h} \bar{u}_{h}-y_{d}, S_{h} \bar{u}-S_{h} \bar{u}\right) .
\end{aligned}
$$

With the help of the Cauchy-Schwarz inequality and (3.2), the last two terms can be estimated by $\mathcal{O}\left(h^{2}\right)$. Thus, all in all we have

$$
\begin{aligned}
\nu\left\|\bar{u}-\bar{u}_{h}\right\|_{L^{2}(\Omega)}^{2}+\left\|S \bar{u}-S_{h} \bar{u}_{h}\right\|_{L^{2}(\Omega)}^{2}+\mathcal{O}\left(h^{2}\right) & \leq\left(\bar{p}+\nu \bar{u}, u_{\sigma}^{h}-\bar{u}\right)+\left(\bar{p}_{h}+\nu \bar{u}_{h}, u_{\delta}-\bar{u}_{h}\right) \\
& \leq c\left(\left\|u_{\sigma}^{h}-\bar{u}\right\|+\left\|u_{\delta}-\bar{u}\right\|\right),
\end{aligned}
$$

while the last step can of course only be taken under the restriction that the chosen norms permit such an estimate. At this stage, we have also tacitly assumed that $p_{h}, y_{h}, u_{h}$ are all uniformly bounded. This, however, is easy to check given the continuity of $S_{h}$ and $S_{h}^{*}$, the approximation results (3.2) and the fact that $a \leq u_{h}(x) \leq b$ $\forall h>0$.

The estimate above demonstrates that the question of proving the rate of convergence boils down to finding test functions $u_{\delta}$ and $u_{\sigma}^{h}$ close to the continuous and discrete solutions respectively. We now need to construct such functions. To do this, though, we cannot do without some auxiliary approximation results, which are discussed in a general setting in the next chapter.

\section{Approximation Results}

We will discuss the ensuing results in a very general setting, applying them later to the control-to-state mapping $S$ and the difference $u-P_{h} u$, where $P_{h}$ denotes the $L^{2}$-projection on the space of piecewise constant functions, interpreted as a linear functional on certain suitable spaces. As a consequence, let us merely assume that there is a mapping $S$ and a function $w$ which possess the following properties:

$$
\begin{aligned}
& S \in \mathcal{L}\left(W^{1, p^{\prime}}(\Omega)^{*}, W^{1, p}(\Omega)\right), 2 \leq p<3 \\
& S \in \mathcal{L}\left(L^{p}(\Omega), W^{2, p}(\Omega)\right), 2 \leq p<3 .
\end{aligned}
$$

For $w$ we assume that $w \in L^{\infty}(\Omega)$. We observe that $w$ naturally defines a linear functional on a suitable space $V$ by

$$
w(v):=(w, v)_{L^{2}(\Omega)} \quad v \in V .
$$


For convenience, we will use $w$ as a function and $w$ as a functional interchangeably. $w$ possesses the following properties:

$$
\begin{aligned}
& w \in L^{p}(\Omega)^{*}=L^{p^{\prime}}(\Omega) \\
& w \in W^{1, p^{\prime}}(\Omega)^{*}, \quad 1 \leq p<\infty .
\end{aligned}
$$

Besides, we postulate an orthogonality property for the space of piecewise constant functions $U_{h} \subset L^{2}(\Omega)$

$$
\left(w, u_{h}\right)_{L^{2}(\Omega)}=0 \quad \forall u_{h} \in U_{h} .
$$

What we are interested in is an estimate of the type:

$$
\|S w\|_{L^{\infty}(\Omega)} \leq c\|w\|_{X},
$$

i.e. a continuity property of $S$ as a mapping from $X$ to $L^{\infty}(\Omega)$, where $X$ is a space between $W^{1, p^{\prime}}(\Omega)^{*}$ and $L^{p}(\Omega)^{*}$, as close to - and thus as 'weak' as - the former as possible.

To construct such intermediate spaces $X$, we employ the J-method of interpolation, see [1], Chapter 7, sticking also to the notation introduced there: for two Banach spaces $X, Y$ satisfying $Y \hookrightarrow X$ we denote the resulting interpolation space $Z$ by:

$$
Z=(X, Y)_{\theta, q ; J} \quad 0 \leq \theta \leq 1,1 \leq q \leq \infty .
$$

In a way, however, we will start at the other end, i.e. we are going to tackle the question of which space between $W^{1, p}(\Omega)$ and $W^{2, p}(\Omega)$ is regular enough to allow for an embedding into $L^{\infty}(\Omega)$. To construct such spaces, we will employ the technique of interpolation spaces.

First of all, from [1], Theorem 7.34 we know that

$$
B^{s, p, 1}(\Omega)=\left(W^{1, p}(\Omega), W^{2, p}(\Omega)\right)_{\theta, 1 ; J} \hookrightarrow L^{\infty}(\Omega),
$$

where $s=1+\theta=\frac{3}{p}$ and $B^{s, p, 1}(\Omega)$ denotes a Besov space, compare [1].

Using interpolation space properties (see [1]) and (5.1), we obtain continuity of the mapping

$$
S: X=\left(W^{1, p^{\prime}}(\Omega)^{*}, L^{p}(\Omega)\right)_{\theta, 1 ; J} \mapsto B^{s, p, 1}(\Omega) \hookrightarrow L^{\infty}(\Omega) .
$$

To arrive at an estimate similar to (5.6), though, we need to get a representation of $X$ as (at least) a subspace of some dual space of a space lying between $W^{1, p^{\prime}}(\Omega)$ and $L^{p}(\Omega)$.

In order to do so we first make use of the fact that in some sense $X \subset X^{* *}$. Employing once again standard interpolation space properties (see [4], Chap. 3), we can write:

$$
\begin{aligned}
X \subset X^{* *} & =\left(W^{1, p^{\prime}}(\Omega)^{*}, L^{p}(\Omega)\right)_{\theta, 1 ; J}^{* *} \\
& =\left(L^{p^{\prime}}(\Omega)^{*}, W^{1, p^{\prime}}(\Omega)^{*}\right)_{1-\theta, 1 ; J}^{* *} \\
& =\left(L^{p^{\prime}}(\Omega), W^{1, p^{\prime}}(\Omega)\right)_{1-\theta, \infty ; J}^{*} \\
& =B^{2-3 / p, p^{\prime}, \infty}(\Omega)^{*} .
\end{aligned}
$$

Thus, all in all, we can estimate

$$
\begin{aligned}
\|S w\|_{L^{\infty}(\Omega)} & \leq c\|w\|_{B^{2-3 / p, p^{\prime}, \infty}(\Omega)^{*}} \\
& \leq c\|w\|_{L^{p^{\prime}(\Omega)}}^{3 / p-1}\|w\|_{W^{1, p^{\prime}}(\Omega)^{*}}^{2-3 / p}
\end{aligned}
$$

For $p>3$ the situation is considerably easier, since we can use the standard embedding $W^{1, p}(\Omega) \hookrightarrow C(\bar{\Omega})$, which helps us to obtain:

$$
\|S w\|_{L^{\infty}(\Omega)} \leq c\|S w\|_{W^{1, p}(\Omega)} \leq c\|w\|_{W^{1, p^{\prime}}(\Omega)^{*}} .
$$


By means of (5.3) and the Hölder-inequality we can further estimate

$$
\begin{aligned}
\|w\|_{W^{1, p^{\prime}}(\Omega)^{*}} & =\sup _{\|v\|_{W 1, p^{\prime}(\Omega)}=1}|(w, v)| \\
& \leq \sup _{\|v\|_{W^{1, p^{\prime}(\Omega)}}=1}\left\{\left|\left(w, v-P_{h} v\right)_{L^{2}(\Omega)}\right|\right\} \\
& \leq\|w\|_{L^{p}(\Omega)} \sup _{\|v\|_{W^{1, p^{\prime}}(\Omega)}=1}\left\{\left\|v-P_{h} v\right\|_{L^{p^{\prime}}(\Omega)}\right\} \\
& \leq c h\|w\|_{L^{p}(\Omega)},
\end{aligned}
$$

where we have used (3.4). $P_{h}$ denotes the $L^{2}$-projection on $U_{h}$.

We will summarise these results in a short lemma:

Lemma 5.1. Let $w$ and $S$ satisfy all the above assumptions. Then for $p>3$ we have

$$
\|S w\|_{L^{\infty}(\Omega)} \leq c \sup _{\|v\|_{W^{1, p^{\prime}}(\Omega)}=1}\left\{\|w\|_{L^{p^{\prime}}(\Omega)}\left\|v-P_{h} v\right\|_{L^{p}(\Omega)}\right\} \leq \operatorname{ch}\|w\|_{L^{p^{\prime}}(\Omega)}
$$

where $P_{h}$ again denotes the $L^{2}$-projection on $U_{h}$.

For $2 \leq p<3$ the estimate

$$
\|S w\|_{L^{\infty}(\Omega)} \leq c\|w\|_{L^{p^{\prime}(\Omega)}}^{3 / p-1}\|w\|_{W^{1, p^{\prime}}(\Omega)^{*}}^{2-3 / p} .
$$

holds.

Proof. We refer to (5.5) and (5.6) for (5.7) and (5.4) for (5.8).

We can now tackle the actual construction of the test functions for (2.1) and (3.1).

\section{Construction of test functions}

Before we delve into the proofs and the associated technicalities, we will outline our strategy and the problems that go along with it.

For the purely control-constrained case one would typically choose $u_{\delta}=\bar{u}_{h}$ and $u_{\sigma}^{h}=P_{h} \bar{u}$. These two simple choices are admissible, because in this case $U_{a d}^{h} \subset U_{a d}$. Since, however, we have included the state constraint into our admissible sets, $U_{a d}^{h}$ is no longer a subset of $U_{a d}$. That's why such relatively straightforward choices of test functions as the projection of $\bar{u}$ are no longer applicable. Instead, we will have to deal with questions such as uniform bounds on the violation of the state constraint by $S \bar{u}_{h}$ and $S P_{h} \bar{u}$.

Let us now commence with the construction of $u_{\delta} \in U_{a d}$.

\subsection{Continuous test function}

We will choose $u_{\delta}$ as a convex linear combination, i.e.

$$
u_{\delta}=(1-\delta) \bar{u}_{h}+\delta u_{s} .
$$

Choosing a convex linear combination ensures that we satisfy the control constraints, while the $u_{s}$ in some sense constitutes a 'movement into the right direction', namely one towards feasibility for $u_{\delta}$ with regard to the state constraint. The ensuing lemma demonstrates that this approach is constructive.

Lemma 6.1. There exist constants $\delta$ and $K$ independent of $u, h$ with $\delta=K h^{2}|\ln h|^{2}$ and $u_{\delta}:=(1-\delta) \bar{u}_{h}+\delta u_{s} \in$ $U_{\text {ad }}$ for all $h \leq h_{1}<1$. Furthermore:

$$
\left\|\bar{u}-u_{\delta}\right\|_{L^{2}(\Omega)} \leq c h^{2}|\ln h|^{2}\left(\left\|u_{\delta}\right\|_{L^{2}(\Omega)}+\|\bar{u}\|_{L^{2}(\Omega)}\right) .
$$


Proof. Let us first verify that $u_{\delta}$ satisfies the pointwise state constraint for some $\delta \leq 1$.

$$
\begin{aligned}
-S u_{\delta} & =-\left((1-\delta) S \bar{u}_{h}+\delta S u_{s}\right) \\
& =-\left((1-\delta) S \bar{u}_{h}-(1-\delta) S_{h} \bar{u}_{h}+(1-\delta) S_{h} \bar{u}_{h}+\delta S u_{s}\right) \\
& =-\left((1-\delta)\left(S-S_{h}\right) \bar{u}_{h}+(1-\delta) S_{h} \bar{u}_{h}+\delta S u_{s}\right) \\
& =\left((1-\delta)\left(S_{h}-S\right) \bar{u}_{h}-(1-\delta) S_{h} \bar{u}_{h}-\delta S u_{s}\right) \\
& \leq(1-\delta)\left\|\left(S-S_{h}\right) \bar{u}_{h}\right\|_{L^{\infty}(\Omega)}-(1-\delta) y_{c}-\delta\left(y_{c}+\tau\right) \\
& \leq c h^{2}|\ln h|^{2}-y_{c}-\delta \tau .
\end{aligned}
$$

In the last line we used the third inequality in (3.2). Multiplying the inequality by -1 yields

$$
S u_{\delta} \geq y_{c}+\delta \tau-c h^{2}|\ln h|^{2} .
$$

If we choose $\delta$ in the above equation so that it satisfies $\delta \tau=c h^{2}|\ln h|^{2}$, we obtain $\delta=K h^{2}|\ln h|^{2}$, where $K=\frac{c}{\tau}$ is independent of $u, h$, as well as $S u_{\delta} \geq y_{c}$.

A short computation yields that $g(h):=h^{2}|\ln h|^{2}$ is monotonically decreasing for all $0<h \leq h_{d}<1$. By choosing $h_{1}$ as $h_{1}:=\max _{h \leq h_{d}}\left\{: h^{2}|\ln h|^{2} \leq \frac{\tau}{c}\right\}$, we obtain $\delta \leq 1$. This, in turn, guarantees that $u_{\delta} \in U_{a d}^{h}$. The estimate (6.1) is straightforwardly calculated.

Let us point out that in the 'closeness' estimate (6.1) we are restricted by the approximation order coming from the finite element discretisation of the PDE. In a way, this is natural, because due to (3.2) the pointwise error $\left|(S \bar{u})(x)-\left(S_{h} \bar{u}\right)(x)\right|$ cannot exceed $h^{2}|\ln h|^{2}$, which in turn means that $S_{h} \bar{u}$ cannot violate the state constraints more than this amount, since $\bar{u}$ is feasible.

We can now turn to the construction of the test function $u_{\sigma}^{h}$, which will turn out to be more difficult.

\subsection{Discrete test function}

Again we choose $u_{\sigma}^{h}$ as a convex linear combination in a similar vein to $u_{\delta}$, i.e.:

$$
u_{\sigma}^{h}=(1-\sigma) P_{h} \bar{u}+\sigma P_{h} u_{s} .
$$

First of all, we have to check that $P_{h} u_{s}$ is a 'Slater-point' with regard to the discrete admissible set, because again we have to make sure that we move in the right direction, i.e. towards feasibility:

Lemma 6.2. Suppose $u_{s}$ satisfies the Slater-assumption. Then there exists a $\tau_{0}>0$ with $S_{h} P_{h} u_{s} \geq y_{c}+\tau_{0}$ for all $0<h \leq h_{2}<1$. In addition $a \leq P_{h} u_{s}(x) \leq b$ a.e. in $\Omega$.

Proof. That $P_{h} u_{s}$ satisfies the box constraints is a direct consequence of the fact that the $L^{2}$-projection on $U_{h}$ is given by the element-wise mean values on all tetrahedras $T \in \mathcal{T}_{h}$.

For the Slater-property we estimate in the following fashion:

$$
\begin{aligned}
-S_{h} P_{h} u_{s} & =-\left(S u_{s}+S P_{h} u_{s}-S u_{s}+S_{h} P_{h} u_{s}-S P_{h} u_{s}\right) \\
& =-\left(S\left(P_{h} u_{s}-u_{s}\right)+\left(S-S_{h}\right) P_{h} u_{s}+S u_{s}\right) \\
& =\left(S\left(u_{s}-P_{h} u_{s}\right)+\left(S_{h}-S\right) P_{h} u_{s}-S u_{s}\right) \\
& \leq\|S\|_{\mathcal{L}\left(L^{2}(\Omega), L^{\infty}(\Omega)\right)}\left\|u_{s}-P_{h} u_{s}\right\|_{L^{2}(\Omega)}+\left\|\left(S_{h}-S\right) P_{h} u_{s}\right\|_{L^{\infty}(\Omega)}-y_{c}-\tau \\
& \leq c_{1} h\|S\|\left\|u_{s}\right\|_{H^{1}(\Omega)}+c_{2} h^{2}|\ln h|^{2}-y_{c}-\tau .
\end{aligned}
$$

In the last line we have used estimate (3.2) and the one for the $L^{2}$-Projection on the space of piecewise constant functions (3.3). We observe that one can find a suitable $h_{d}$ so that the function $g(h):=h^{2}|\ln h|^{2}$ is 
monotonically decreasing for all $0<h \leq h_{d}<1$. If we now choose $h_{2}$ as $h_{2}:=\max _{h \leq h_{d}}\left\{g(h) \leq \frac{c_{1}}{c_{2}}\|S\|\left\|u_{s}\right\|_{H^{1}(\Omega)}\right\}$, we can conclude:

$$
-S_{h} P_{h} u_{s} \leq c h-y_{c}-\tau
$$

for some generic constant $\mathrm{c}$ independent of $u, h$.

By defining $\tau_{0}=\tau-\tilde{\tau_{0}}$ with $\tilde{\tau_{0}}:=\max _{h \leq h_{2}}\left\{h: y_{c}+\tau-h>0\right\}$, we get

$$
S_{h} P_{h} u_{s} \geq y_{c}+\tau_{0}
$$

after multiplying (6.2) by -1 .

What we would now like to obtain is an error bound on the maximal pointwise violation of the state constraint by $S_{h} P_{h} \bar{u}$, because such a bound determines how far we have to move away from $P_{h} \bar{u}$ in the direction of $P_{h} u_{s}$ when it comes to constructing $u_{\sigma}^{h}$. In the end, as we have already observed in the preceding section, this bound naturally leads to one for $\sigma$. The optimal result in a certain sense would be $\sigma \approx \delta$. As we will see, however, this is something we will not be able to achieve: $\sigma$ will behave like $\mathcal{O}\left(h^{3-3 / p}\right), 2 \leq p<3$ depending on the regularity of $\bar{u} \in W^{1, p}(\Omega)$. For $p>3$, however, $\sigma \approx \delta$. Let us now touch a bit on the reason for this loss of order for lower regularity of $\bar{u}$ by estimating the maximal violation of $S P_{h} u_{s}$ of the state constraint.

$$
\begin{aligned}
\left(y_{c}-S_{h} P_{h} \bar{u}\right)_{+} & =\left(y_{c}-S \bar{u}+S \bar{u}-S_{h} P_{h} \bar{u}\right)_{+} \\
& \leq\left(S \bar{u}-S_{h} P_{h} \bar{u}\right)_{+} \\
& =\left(S \bar{u}-S P_{h} \bar{u}+S P_{h} \bar{u}-S_{h} P_{h} \bar{u}\right)_{+} \\
& \leq\left(S\left(\bar{u}-P_{h} \bar{u}\right)\right)_{+}+\left(\left(S-S_{h}\right) P_{h} \bar{u}\right)_{+} \\
& \leq\left\|S\left(\bar{u}-P_{h} \bar{u}\right)\right\|_{L^{\infty}(\Omega)}+\left\|\left(S-S_{h}\right) P_{h} \bar{u}\right\|_{L^{\infty}(\Omega)} .
\end{aligned}
$$

While the last term can again be dealt with using (3.2), it is the first term that is difficult to control. Despite having (3.4) we are unable to employ this estimate directly, because $S$ is not continuous from $W^{1 \cdot p^{\prime}}(\Omega)^{*}$ to $L^{\infty}(\Omega) . p<3$. Continuity, though, is something we want to take advantage of, which in turn leaves us only with the possibility of choosing a 'better' space.

At this stage we can apply the results of Section 5. First of all, we remark that $w=u-P_{h} u$ and $S$ as the control-to-state mapping fulfil all the requirements on $S$ and $w$ from this section. In the case of $w$ this is easy to check, while for $S$ it should perhaps be remarked that $S \in \mathcal{L}\left(L^{p}(\Omega), W^{2, p}(\Omega)\right)$ because of [14], Theorem 2.4.2.5, while $S \in \mathcal{L}\left(W^{1, p^{\prime}}(\Omega)^{*}, W^{1, p}(\Omega)\right), 2 \leq p<\infty$ is a result that can be proved by interpolation space techniques and [18] as well as [29] or [2].

We can now apply Lemma 5.1, which for $2 \leq p<3$ yields (compare (5.7) and (5.4)):

$$
\begin{aligned}
\left\|S\left(\bar{u}-P_{h} \bar{u}\right)\right\|_{L^{\infty}(\Omega)} & \leq c\left\|\bar{u}-P_{h} \bar{u}\right\|_{L^{p^{\prime}}(\Omega)}^{3 / p-1}\left\|\bar{u}-P_{h} \bar{u}\right\|_{W^{1, p^{\prime}}(\Omega)^{*}}^{2-3 / p} \\
& \leq h^{3-3 / p}\|\bar{u}\|_{W^{1, p}(\Omega)} .
\end{aligned}
$$

In the last line we have also used (3.3) and (3.4).

In the case of $p>3$ we can also make use of Lemma 5.1, and obtain:

$$
\begin{aligned}
\left\|S\left(\bar{u}-P_{h} u\right)\right\|_{L^{\infty}(\Omega)} & \leq c\left\|\bar{u}-P_{h} \bar{u}\right\|_{L^{p}(\Omega)}\left\|\bar{u}-P_{h} \bar{u}\right\|_{L^{p^{\prime}}(\Omega)} \\
& \leq h^{2} c\|\bar{u}\|_{W^{1, p}(\Omega)} .
\end{aligned}
$$

Again, we have employed (3.3) and (3.4). 
Having ascertained these estimates, we can now prove a lemma similar to Lemma 6.1:

Lemma 6.3. There exists $\sigma$ and a constant $K$ independent of $u, h$ so that for $\bar{u} \in W^{1, p}(\Omega)$ and $2 \leq p<3$ $\sigma=K h^{3-3 / p}\|\bar{u}\|_{W^{1, p}(\Omega)}$ and $u_{\sigma}^{h}=(1-\sigma) P_{h} \bar{u}+\sigma P_{h} u_{s} \in U_{a d}^{h}$ for all $0<h \leq h_{3}<1$. Furthermore,

$$
\left\|\bar{u}-u_{\sigma}^{h}\right\|_{B^{2-3 / p, p^{\prime}, \infty}(\Omega)^{*}} \leq c h^{3-3 / p}\left(\|\bar{u}\|_{W^{1, p}(\Omega)}+\left\|u_{s}\right\|_{L^{2}(\Omega)}\right) .
$$

In case $p>3$ we obtain $\sigma=K h^{2}|\ln h|^{2}\|\bar{u}\|_{W^{1, p}(\Omega)}$ and $u_{\sigma}^{h}=(1-\sigma) P_{h} \bar{u}+\sigma P_{h} u_{s} \in U_{\text {ad }}^{h}$ for all $0 h \leq h_{4}<1$. Besides, there holds:

$$
\left\|\bar{u}-u_{\sigma}^{h}\right\|_{W^{1, p^{\prime}}(\Omega)^{*}} \leq c h^{2}|\ln h|^{2}\left(\|\bar{u}\|_{W^{1, p}(\Omega)}+\left\|u_{s}\right\|_{L^{2}(\Omega)}\right) .
$$

Proof. The proof runs more or less on the same lines as that of Lemma 6.1. We restrict ourselves to the case of $2 \leq p<3$, the case of $p>3$ can be examined completely analogously.

We estimate

$$
\begin{aligned}
-S_{h} u_{\sigma}^{h} & =-\left((1-\sigma) S_{h} P_{h} \bar{u}+\sigma S_{h} P_{h} u_{s}\right) \\
& =-\left((1-\sigma) S_{h} P_{h} \bar{u}-(1-\sigma) S P_{h} \bar{u}+(1-\sigma) S P_{h} \bar{u}+\sigma S_{h} P_{h} u_{s}\right) \\
& =-\left((1-\sigma)\left(S_{h}-S\right) P_{h} \bar{u}+(1-\sigma) S P_{h} \bar{u}+(1-\sigma) S \bar{u}-(1-\sigma) S \bar{u}+\sigma S_{h} P_{h} u_{s}\right) \\
& =(1-\sigma)\left(S-S_{h}\right) P_{h} \bar{u}+(1-\sigma) S\left(\bar{u}-P_{h} \bar{u}\right)-(1-\sigma) S \bar{u}-\sigma S_{h} P_{h} u_{s} .
\end{aligned}
$$

We continue using (3.2) and (6.3):

$$
\begin{aligned}
-S_{h} u_{\sigma}^{h} & \leq c h^{2}|\ln h|^{2}+(1-\sigma)\|S\| h^{3-3 / p}\|\bar{u}\|_{W^{1, p}(\Omega)}-(1-\sigma) y_{c}-\sigma\left(y_{c}+\tau_{0}\right) \\
& \leq c h^{3-3 / p}-y_{c}-\sigma \tau_{0} .
\end{aligned}
$$

Again, we have to adjust $h$, but since the involved steps do not differ from those taken in the proof of Lemma 6.1, they need not be repeated here.

The estimate (6.5) is a straightforward calculation, where we also use some imbedding properties of interpolation spaces.

These results enable us to continue where we left off in (4.1).

\section{The RATE OF CONVERGENCE}

We have now collected all the necessary ingredients to derive the convergence rate of $\mathcal{O}\left(h^{3 / 2-3 / 2 p}\right), p<3$, or, in case of higher regularity, that of $\mathcal{O}(h|\ln h|)$.

Theorem 7.1 (the rate of convergence). There exists a constant $c$ independent of $y, u, h$ so that in case $u \in$ $W^{1, p}(\Omega), 2 \leq p<3$, the following estimate holds for all $0<h \leq h_{4}<1$ :

$$
\nu\left\|\bar{u}-\bar{u}_{h}\right\|_{L^{2}(\Omega)}+\left\|\bar{y}-\bar{y}_{h}\right\|_{L^{2}(\Omega)} \leq c h^{3 / 2-3 / 2 p}\|\bar{u}\|_{W^{1, p}(\Omega)} .
$$

If $u \in W^{1, p}(\Omega)$ with $p>3$, there exists a constant $c$ independent of $y, u, h$ so that for all $0<h \leq h_{5}<1$

$$
\nu\left\|\bar{u}-\bar{u}_{h}\right\|_{L^{2}(\Omega)}+\left\|\bar{y}-\bar{y}_{h}\right\|_{L^{2}(\Omega)} \leq c h|\ln h|\|\bar{u}\|_{W^{1, p}(\Omega)} .
$$

Proof. Let us recall the last estimate in Section 4, i.e. (4.1).

$$
\nu\left\|\bar{u}-\bar{u}_{h}\right\|_{L^{2}(\Omega)}^{2}+\left\|S \bar{u}-S_{h} \bar{u}_{h}\right\|_{L^{2}(\Omega)}^{2}+\mathcal{O}\left(h^{2}\right) \leq\left(\bar{p}+\nu u, u_{\sigma}^{h}-\bar{u}\right)+\left(\bar{p}_{h}+\nu \bar{u}_{h}, u_{\delta}-\bar{u}_{h}\right) .
$$

What is now left to do is to estimate the two terms on the right. Naturally, we want to employ the estimates (6.1), (6.5) and (6.6). 
We can treat the first with the Cauchy-Schwarz inequality, which, together with (6.1) and the uniform boundedness of $\bar{p}_{h}$ and $\bar{u}_{h}$ in $L^{2}(\Omega)$, yields:

$$
\left(\bar{p}_{h}+\nu \bar{u}_{h}, u_{\delta}-\bar{u}_{h}\right) \leq c\left\|\bar{u}-u_{\delta}\right\|_{L^{2}(\Omega)} \leq c h^{2}|\ln h|^{2} .
$$

The second one can be estimated by:

$$
\left(\bar{p}+\nu \bar{u}, u_{\sigma}^{h}-\bar{u}\right) \leq c\left\|\bar{u}-u_{\sigma}^{h}\right\|_{Y},
$$

where $Y=B^{2-3 / p, p^{\prime}, \infty}(\Omega)$ for $2 \leq p<3$ and $Y=W^{1, p^{\prime}}(\Omega)$ for $p>3$. Let us now check that such an estimate is indeed admissible: since $u_{\sigma}^{h}, \bar{u} \in L^{\infty}(\Omega),\left(\cdot, u_{\sigma}^{h}-\bar{u}\right)$ is a linear functional on all $L^{p}(\Omega)$ and $W^{1, p}(\Omega)$, $1 \leq p \leq \infty$. Thus, $u_{\sigma}^{h}-\bar{u} \in W^{1, p^{\prime}}(\Omega)^{*}, p>3$, and using interpolation space properties we can conclude that $u_{\sigma}^{h}-\bar{u} \in B^{2-3 / p, p^{\prime}, \infty}(\Omega)^{*}, 2 \leq p<3$. Thus (7.3) is nothing but a continuity estimate.

We can now employ (6.5) and (6.6) and estimate

$$
\begin{aligned}
\left(\bar{p}+\nu \bar{u}, u_{\sigma}^{h}-\bar{u}\right) & \leq\|\bar{p}+\nu \bar{u}\|_{B^{2-3 / p, p^{\prime}, \infty}(\Omega)}\left\|u_{\sigma}^{h}-\bar{u}\right\|_{B^{2-3 / p, p^{\prime}, \infty}(\Omega)^{*}} \\
& \leq c h^{3-3 / p}
\end{aligned}
$$

for $p<3$. For $p>3$ there holds:

$$
\begin{aligned}
\left(\bar{p}+\nu \bar{u}, u_{\sigma}^{h}-\bar{u}\right) & \leq\|\bar{p}+\nu \bar{u}\|_{W^{1, p^{\prime}}(\Omega)}\left\|u_{\sigma}^{h}-\bar{u}\right\|_{W^{1, p^{\prime}}(\Omega)^{*}} \\
& \leq c h^{2} .
\end{aligned}
$$

All in all, we now have

$$
\nu\left\|\bar{u}-\bar{u}_{h}\right\|_{L^{2}(\Omega)}^{2}+\left\|S \bar{u}-S_{h} \bar{u}_{h}\right\|_{L^{2}(\Omega)}^{2} \leq c h^{3-3 / p}
$$

for $p<3$, and for $p>3$ :

$$
\nu\left\|\bar{u}-\bar{u}_{h}\right\|_{L^{2}(\Omega)}^{2}+\left\|S \bar{u}-S_{h} \bar{u}_{h}\right\|_{L^{2}(\Omega)}^{2} \leq c h^{2}|\ln h|^{2} .
$$

Drawing the square root yields (7.1) and (7.2) respectively.

\section{NUMERICAL EXPERIMENTS}

To compute an approximative solution of problem $(\mathrm{P})$, we employed a virtual control regularisation technique for the state constraint, turning it into a purely control-constrained problem. For more details about this technique we refer to e.g. [9]. This was then solved with a primal-dual active set strategy, which has been treated among others in $[5,15,17]$.

All computations were done with ALBERTA, see [26].

For an analytic solution we slightly modified the PDE in (P), including an additional function $f$ on the right-hand side. This does not affect the analysis we undertook in the preceding sections.

$$
\begin{aligned}
-\Delta y & =u+f & & \text { in } \Omega \\
y & =0 & & \text { on } \Gamma .
\end{aligned}
$$

Let us now come to the details of our example: we took $\Omega$ as the unit ball in $\mathbb{R}^{3}$, set $a=-1$ and $b=1$ and chose the following function:

$$
\begin{aligned}
u(x) & =-\sin \left(\pi|x|^{2}\right) \\
y(x) & =\cos \left(\frac{\pi}{2}|x|^{2}\right) \\
y_{d}(x) & =-4 \pi^{2}|x|^{2} \sin \left(\pi|x|^{2}\right)+6 \pi \cos \left(\pi|x|^{2}\right)+\cos \left(\frac{\pi}{2}|x|^{2}\right) \\
f(x) & =3 \pi \sin \left(\frac{\pi}{2}|x|^{2}\right)+\pi^{2}|x|^{2} \cos \left(\frac{\pi}{2}|x|^{2}\right)+\sin \left(\pi|x|^{2}\right)
\end{aligned}
$$


TABLE 1. $L^{2}$-errors control and state.

\begin{tabular}{|c|c|c|}
\hline$h_{T}$ & $\left\|u-u_{h, \varepsilon}\right\|$ & $\left\|y-y_{h, \varepsilon}\right\|$ \\
\hline 0.043335 & 0.348817 & 0.064930 \\
\hline 0.034395 & 0.270962 & 0.034141 \\
\hline 0.027299 & 0.222425 & 0.029520 \\
\hline 0.021668 & 0.167978 & 0.016696 \\
\hline 0.017198 & 0.134295 & 0.008157 \\
\hline 0.013650 & 0.111856 & 0.006841 \\
\hline 0.010834 & 0.087764 & 0.003957 \\
\hline 0.008599 & 0.070659 & 0.001721 \\
\hline
\end{tabular}

Comparison of Convergence Errors

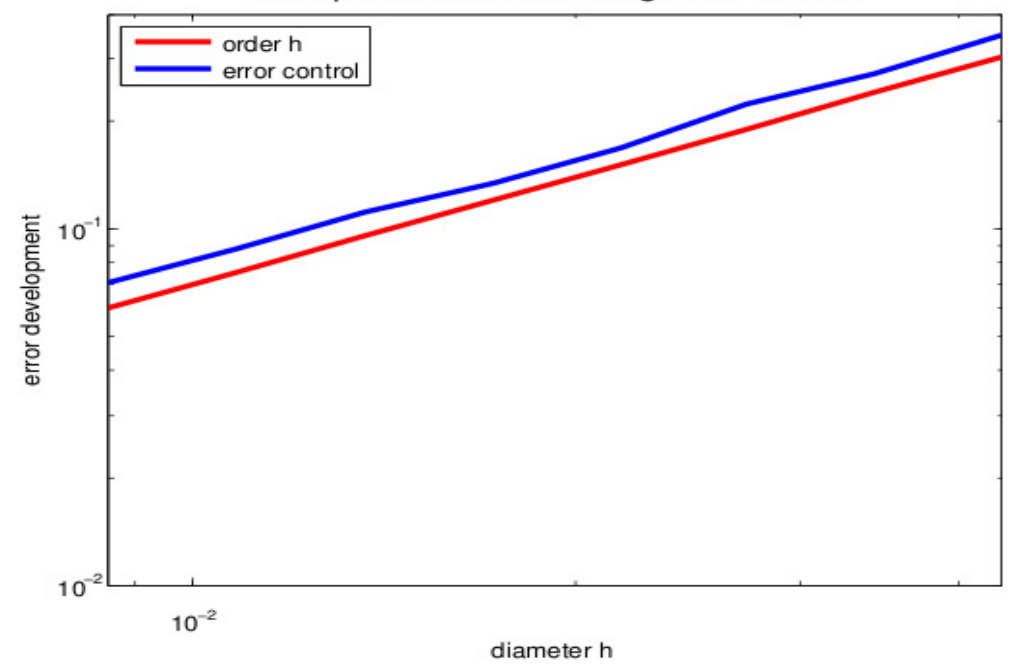

FIGURE 1. $L^{2}$-error behaviour for control.

$y_{c}$ was adjusted to be active on a spherical shell, i.e.:

$$
y_{c}(x)= \begin{cases}y(x) & \text { if }|x| \leq \frac{1}{2} \\ \left(-\frac{4}{3} \cos \left(\frac{\pi}{8}\right)-\frac{40}{3}\right)|x|^{2}+\frac{4}{3} \cos \left(\frac{\pi}{8}\right)+\frac{10}{3} & \text { else. }\end{cases}
$$

The finest grid on which we could compute a solution before we ran into memory trouble consisted of 1, 048, 576 elements. We obtained the following results for the $L^{2}$-errors of the control and the state in the case of $\varepsilon=0.01$, which we list in Table 1.

These errors contain an additional error besides the discretisation error, namely a regularisation error, which has its source in the optimisation method we used. However, tests have shown that the discretisation error dominates.

Graphically, the $L^{2}$-error in the control $u$ is reflected by the 'error control' curve in Figure 1. For a comparison with the theoretical convergence rate $\mathcal{O}(h)$ we have added its curve.

As one can see, our theoretical convergence rate of nearly $h$ is reflected in this example.

\section{Concluding Remarks}

One question that perhaps naturally springs to the mind is whether the result proven in Theorem 7.1 can be extended to a more general setting, i.e. a more general elliptic equation in $(\mathrm{P})$ with different boundary 
conditions, e.g. non-homogeneous Dirichlet conditions, Neumann or Robin boundary conditions. As long as $L^{\infty}$-estimates of the type (3.2) hold - perhaps with different exponents - and the solution to the underlying PDE in (P) belongs to $H^{2}(\Omega)$, our analysis can be easily transferred to the more general setting. For one example of such an $L^{\infty}$-estimate for a more general elliptic operator we refer to [21].

To discretise Neumann boundary conditions given on a smooth boundary one can simply consider the corresponding bilinear form of the weak formulation solely on $\Omega_{h}$. If the data are smooth enough than the standard error estimates in the $H^{1}$ - and $L^{2}$-norm in (3.2) still hold, see e.g. [3].

Besides by enforcing the state constraint $y(x) \geq y_{c}(x)$ only on a domain $\Omega^{\prime}$ with $\Omega^{\prime} \subset \subset \Omega$, i.e. $\bar{\Omega}^{\prime} \subset \Omega$, the $L^{\infty}$-estimate in (3.2) only needs to hold on $\Omega^{\prime}$. That way, one can derive such an estimate employing interior maximum norm estimates, e.g. [24]. For $L^{\infty}(\Omega)$ estimates for Neumann boundary conditions we refer to [23]. Thus, in this setting, convex and polyhedral domains $\Omega$ can be included as they also guarantee global $H^{2}(\Omega)$-regularity of the solution $S u=y$.

For numerical purposes it is often desirable to replace the constraint $y_{c}$ with an interpolant $\mathcal{I}_{h} y_{c}$ in the definition of $U_{a d}^{h}$, e.g. the standard Lagrange interpolant. If we assume that $y_{c} \in W^{2, \infty}(\Omega)$, our results can be transferred in a fairly straightforward way. We refer to the paper by Deckelnick and Hinze [12] for details.

\section{REFERENCES}

[1] R.A. Adams and J.J.F. Fournier, Sobolev spaces. Academic Press, San Diego (2007).

[2] S. Agmon, A. Douglis and L. Nirenberg, Estimates near the boundary for solution of elliptic partial differential equations satisfying general boundary conditions I. Comm. Pure Appl. Math. 12 (1959) 623-727.

[3] J.W. Barrett and C.M. Elliott, A finite-element method for solving elliptic equations with Neumann data on a curved boundary using unfitted meshes. IMA J. Numer. Anal. 4 (1984) 309-325.

[4] J. Bergh and J. Löfström, Interpolation spaces. Springer, Berlin (1976).

[5] M. Bergounioux, K. Ito and K. Kunisch, Primal-dual strategy for constrained optimal control problems. SIAM J. Control Optim. 37 (1999) 1176-1194.

[6] E. Casas, Control of an elliptic problem with pointwise state constraints. SIAM J. Control Optim. 4 (1986) 1309-1322.

[7] E. Casas and F. Tröltzsch, Recent advances in the analysis of pointwise state-constrained elliptic optimal control problems. ESAIM: COCV 16 (2010) 581-600.

[8] S. Cherednichenko and A. Rösch, Error estimates for the regularization of optimal control problems with pointwise control and state constraints. Z. Anal. Anwendungen 27 (2008) 195-212.

[9] S. Cherednichenko, K. Krumbiegel and A. Rösch, Error estimates for the Lavrentiev regularization of elliptic optimal control problems. Inverse Problems 24 (2008).

[10] P.G. Ciarlet, The finite element method for elliptic problems. SIAM Classics In Applied Mathematics, Philadelphia (2002).

[11] J.C. de los Reyes, C. Meyer and B. Vexler, Finite element error analysis for state-constrained optimal control of the Stokes equations. Control and Cybernetics 37 (2008) 251-284.

[12] K. Deckelnick and M. Hinze, Convergence of a finite element approximation to a state constrained elliptic control problem. SIAM J. Numer. Anal. 45 (2007) 1937-1953.

[13] K. Deckelnick and M. Hinze, Numerical analysis of a control and state constrained elliptic control problem with piecewise constant control approximations, in Numerical Mathematics and Advanced Applications, edited by K. Kunisch, G. Of and O. Steinbach, Berlin, Heidelberg, Springer-Verlag (2008) 597-604.

[14] P. Grisvard, Elliptic Problems in Nonsmooth Domains. Pitman, Boston (1985).

[15] M. Hintermüller, K. Ito and K. Kunisch, The primal-dual active set strategy as a semi-smooth Newton method. SIAM J. Optim. 13 (2003) 865-888.

[16] M. Hinze, R. Pinnau, M. Ulbrich and S. Ulbrich, Optimization with PDE Constraints. Springer-Verlag, Berlin (2009).

[17] K. Kunisch and A. Rösch, Primal-dual active set strategy for a general class of constrained optimal control problems. SIAM J. Optim. 13 (2002) 321-334.

[18] C. Meyer, Error estimates for the finite-element approximation of an elliptic control problem with pointwise state and control constraints. Control and Cybernetics 37 (2008) 51-85.

[19] C. Meyer, A. Rösch and F. Tröltzsch, Optimal control of PDEs with regularized pointwise state constraints. Comput. Optim. Appl. 33 (2006) 209-228.

[20] P. Neittaanmaki, J. Sprekels and D. Tiba, Optimization of Elliptic Systems. Springer-Verlag, New York (2006).

[21] R. Rannacher, Zur $L_{\infty}$-Konvergenz linearer finiter elemente beim Dirichlet-problem. Math. Z. 149 (1976) 69-77.

[22] A. Rösch and F. Tröltzsch, Existence of regular Lagrange multipliers for elliptic optimal control problem with pointwise control-state constraints. SIAM J. Optim. 45 (2006) 548-564. 
[23] A.H. Schatz, Pointwise error estimates and asymptotic error expansion inequalities for the finite element method on irregular grids. I: Global estimates. Math. Comput. 67 (1998) 877-899.

[24] A.H. Schatz and L.B. Wahlbin, Interior maximum norm estimates for the finite element method. Math. Comput. 31 (1977) $414-442$.

[25] A.H. Schatz and L.B. Wahlbin, On the quasi-optimality in $L_{\infty}$ of the $\stackrel{\circ}{ }^{1}$-projection into finite element spaces. Math. Comput. 38 (1982) 1-22.

[26] A. Schmidt and K.G. Siebert, Design of Adaptive Finite Element Software, The Finite Element Toolbox ALBERTA. SpringerVerlag, Berlin (2000).

[27] F. Tröltzsch, Regular Lagrange multipliers for problems with pointwise mixed control-state constraints. SIAM J. Optim. 15 (2005) 616-634.

[28] F. Tröltzsch, Optimal control of partial differential equations. Amer. Math. Soc., Providence, Rhode Island (2010).

[29] D.Z. Zanger, The inhomogeneous Neumann problem in Lipschitz domains. Commun. Partial Differ. Equ. 25 (2000) $1771-1808$. 